\title{
SOLOMON'S CONJECTURES AND THE LOCAL FUNCTIONAL EQUATION FOR ZETA FUNCTIONS OF ORDERS
}

\author{
BY COLIN J. BUSHNELL AND IRVING REINER ${ }^{1}$
}

Let $A$ be a finite dimensional semisimple $K$-algebra, where $K$ is either an algebraic number field or a complete $P$-adic field. Let $R$ be a Dedekind domain with quotient field $K$, and let $\Lambda$ be an $R$-order in $A$. Louis Solomon [3], [4] introduced a zeta function

$$
\zeta_{\Lambda}(s)=\sum_{M \subseteq \Lambda}(\Lambda: M)^{-s}
$$

where $M$ ranges over all full left ideals of $\Lambda$. The series converges for $\operatorname{Re}(s)>1$. Here, $(\Lambda: M)$ is the number of elements in $\Lambda / M$, and $s$ is a complex variable. For the case where $\Lambda=R$, the above is the usual Dedekind zeta function of $R$, namely,

$$
\zeta_{R}(s)=\sum(R: \mathfrak{a})^{-s},
$$

where $\mathfrak{a}$ ranges over all nonzero ideals of $R$.

Let $P$ range over all maximal ideals of $R$, and let $R_{P}, A_{P}$, etc., denote $P$-adic completions. Solomon showed easily that

$$
\zeta_{\Lambda}(s)=\prod_{P} \zeta_{\Lambda_{P}}(s),
$$

and introduced a "global" zeta function $\zeta_{A}(s)$, which depends on $A$ and $R$ but not on $\Lambda$, with the property that the $P$-part of $\zeta_{A}(s)$ coincides with $\zeta_{\Lambda}(s)$ for almost all $P$. (To be explicit, this occurs whenever $A_{P}$ is a direct sum of full matrix algebras over fields, and $\Lambda_{P}$ is a maximal $R_{P}$-order in $A_{P}$.) Solomon's conjectures involve the comparison between $\zeta_{A}(s)$ and $\zeta_{\Lambda}(s)$ at arbitrary $P$ 's.

Let us place the above in the more general setting used by Solomon. Let $L$ be a full $\Lambda$-lattice in an $A$-module $V$, and define

$$
\zeta_{L}(s)=\sum_{M \subseteq L}(L: M)^{-s}
$$

where $M$ ranges over all full $\Lambda$-sublattices of $L$. To define the "global" function $\zeta_{V}(s)$, we start with the Wedderburn decomposition of $A$ :

$$
A=A_{1} \oplus \cdots \oplus A_{r}, \quad A_{i} \cong M_{k_{i}}\left(D_{i}\right), \quad\left(D_{i}: F_{i}\right)=e_{i}^{2}, \quad 1 \leqslant i \leqslant r,
$$

Received by the editors August 20, 1979; presented to the Second International Conference on Representations of Algebras, Ottawa, Canada, August 22, 1979.

AMS (MOS) subject classifications (1970). Primary 12A82, 12B37, 16A18.

${ }^{1}$ The research of this author was supported by the National Science Foundation. 
where $D_{i}$ is a division algebra with center $F_{i}$. Let $W_{i}$ be a simple left $A_{i}$-module, and let $R_{i}$ denote the integral closure of $R$ in $F_{i}$. We may write

$$
V \cong \coprod_{i=1}^{r} w_{i}^{\left(v_{i}\right)}
$$

and now define

where

$$
\zeta_{V}(s)=\prod_{i=1}^{r} \zeta_{W_{i}^{\left(v_{i}\right)}}(s)
$$

$$
\zeta_{W_{i}\left(v_{i}\right)}(s)=\prod_{j=0}^{v_{i} e_{i}-1} \zeta_{R_{i}}\left(k_{i} e_{i} s-j\right)
$$

In particular, setting $n_{i}=k_{i} e_{i}$, we have (by definition)

$$
\zeta_{A}(s)=\prod_{i=1}^{r} \prod_{j=0}^{n_{i}-1} \zeta_{R_{i}}\left(n_{i} s-j\right) .
$$

Now put

$$
\varphi_{P}(s)=\zeta_{L_{P}}(s) / P \text {-part of } \zeta_{V}(s),
$$

where $L$ is a full $\Lambda$-lattice in the $A$-module $V$, and let $p$ be the characteristic of $R / P$. Using an ingenious combinatorial argument, Solomon proved that $\varphi_{p}(s)$ is a rational function of $p^{-s}$, and conjectured that in fact $\varphi_{P}(s) \in \mathbf{Z}\left[p^{-s}\right]$ always. He verified this conjecture whenever $\Lambda_{P}$ is a maximal order, by using the calculations of K. Hey.

Our first main result is

THEOREM 1. Let $R$ be a complete P-adic ring, and let $\Lambda^{\prime}$ be a maximal $R$-order in $A$ containing $\Lambda$. Let $L$ be a full left $\Lambda$-lattice in the A-module $V$. Then

$$
\zeta_{L}(s) / \zeta_{\Lambda^{\prime} L}(s) \in \mathbf{Z}\left[p^{-s}\right]
$$

where $p$ is the characteristic of $R / P$.

In the local case, we have

$$
\zeta_{\Lambda^{\prime} L}(s) / \zeta_{V}(s) \in \mathbf{Z}\left[p^{-s}\right]
$$

by the previous remarks, so as a consequence of Theorem 1 we obtain

THEOREM 2. For each $P, \varphi_{P}(s) \in \mathrm{Z}\left[p^{-s}\right]$.

The method of proof of Theorem 1 (see below) also supports a second conjecture of Solomon's. Let $V$ be a left $A$-module, and let $\left\{L_{1}, \ldots, L_{k}\right\}$ be 
a complete set of nonisomorphic full left $\Lambda$-lattices in $V$. Define

$$
Z_{i j}(s)=\sum_{M}\left(L_{i}: M\right)^{-s}, \quad 1 \leqslant i, j \leqslant k,
$$

where the summation extends over all full $\Lambda$-sublattices $M$ of $L_{i}$ such that $M \cong$ $L_{j}$. Let $\mathrm{B}$ be the $k \times k$ matrix

$$
\mathbf{B}=\left(Z_{i j}(s)\right)_{1 \leqslant i, j \leqslant k} .
$$

Solomon proved that $(\operatorname{det} \mathbf{B})^{-1}$ is a polynomial in $p^{-s}$ with integral coefficients, and conjectured that it can always be expressed as a finite product $\Pi_{i}\left(1-p^{a_{i}-b_{i} s}\right)$, where the $\left\{a_{i}\right\}$ are nonnegative integers and the $\left\{b_{i}\right\}$ positive integers. In support of this conjecture, we have proved

THEOREM 3. The polynomial (det $\mathbf{B})^{-1}$ is a divisor in $\mathbf{Z}\left[p^{-s}\right]$ of a finite product of the above type.

Finally, we turn to the existence of a functional equation for the case where $\Lambda$ is the integral group ring of a finite group $G$ over a complete $P$-adic ring $R$. In this case, the local zeta function $\zeta_{\Lambda}(s)$ has an analytic continuation to a meromorphic function on the whole complex s-plane. Our main result is

THEOREM 4. Let $\Lambda=R G$, where $G$ is a finite group and $R$ is a complete $P$-adic ring. Let $\Lambda^{\prime}$ be a maximal $R$-order in $K G$ containing $\Lambda$. Then there is a functional equation

$$
\zeta_{\Lambda}(s) / \zeta_{\Lambda}(1-s)=\left(\Lambda^{\prime}: \Lambda\right)^{1-2 s} \zeta_{\Lambda^{\prime}}(s) / \zeta_{\Lambda^{\prime}}(1-s)
$$

valid for all s.

Since $\zeta_{\Lambda}$ (s) can be calculated from Hey's formula, the quotient $\zeta_{\Lambda^{\prime}}(s) / \zeta_{\Lambda^{\prime}}(1-s)$ can be given explicitly. Furthermore, the functional equation can be used to give a more precise form of Theorem 1, namely:

COROllary. Keeping the above notation, let $\left(\Lambda^{\prime}: \Lambda\right)=p^{n}$. Then $\zeta_{\Lambda}(s) / \zeta_{\Lambda^{\prime}}(s)$ is a polynomial in $p^{-s}$ with the highest degree term $p^{n(1-2 s)}$.

We prove all of these theorems by applying the methods of Tate's thesis to Solomon's zeta functions. For simplicity of notation, let us restrict our attention to the complete local case, with $L$ chosen equal to $\Lambda$. All of the zeta functions discussed above can be put in the form

$$
c \cdot q^{-s} \int_{A} \times \Phi(x)\|x\|_{A}^{s} d^{\times} x
$$

where $c$ and $q$ are constants, $\Phi$ is a function on $A$ which is locally constant of 
compact support, $A^{\times}$is the group of units of $A, d^{\times} x$ is a Haar measure on $A^{\times}$, and the map $x \mapsto\|x\|_{A}$ is the homomorphism from $A^{\times}$to the group of positive real numbers, determined by the formula $\|x\|_{A}=(\Lambda: \Lambda x)^{-1}$ whenever $x \in \Lambda$.

For example, given a full left $\Lambda$-lattice $M$ in $A$, we can form

$$
Z_{\Lambda}(M ; s)=\sum_{\substack{N \subseteq \Lambda \\ N \cong M}}(\Lambda: N)^{-s} .
$$

Then we get $\zeta_{\Lambda}(s)=\Sigma_{M} Z_{M}(M ; s)$, for $M$ ranging over some finite set. Furthermore,

$$
Z_{\Lambda}(M ; s)=c^{-1}(\Lambda: M)^{-s} \int_{A} \times \Phi(x)\|x\|_{A}^{s} d^{\times} x,
$$

where $\Phi$ is defined by $\Phi(x)=1$ if $M x \subseteq \Lambda$, and $\Phi(x)=0$ otherwise. The constant $c$ is the measure, relative to $d^{\times} x$, of the subgroup Aut ${ }_{\Lambda} M$ of $A$. This formula leads to a corresponding expression for $\zeta_{\Lambda}(s)$ as an integral.

The core of the proof of Theorem 1 consists in showing that

$$
\left\{\zeta_{\Lambda^{\prime}}(s)\right\}^{-1} \int_{A} \times \Phi(x)\|x\|_{A}^{s} d^{\times} x \in \mathrm{C}\left[p^{-s}, p^{s}\right]
$$

for any locally constant $\Phi$ of compact support on $A$, and any maximal order $\Lambda^{\prime}$ in $A$. Using the obvious fact that $Z_{\Lambda}(M ; s) \in \mathbf{Z}\left[\left[p^{-s}\right]\right]$, it follows straightaway that

$$
Z_{\Lambda}(M ; s) / \zeta_{\Lambda^{\prime}}(s) \in \mathbf{Z}\left[p^{-s}\right]
$$

for each $M$. This implies Theorem 1 .

For the functions $\Phi$ as above, we put

$$
Z(\Phi ; s)=\int_{A} \times \Phi(x)\|x\|_{A}^{s} d^{\times} x
$$

This zeta integral converges and defines a holomorphic function of the complex variable $s$ in some half-plane $\operatorname{Re} s>\alpha$, and admits analytic continuation to a meromorphic function of $s$. Further, let $\hat{\Phi}$ denote the Fourier transform of $\Phi$, taken in some suitable manner. Then the quotient $Z(\Phi ; s) / Z(\hat{\Phi} ; 1-s)$ is independent of $\Phi$. These facts are easily deduced from the functional equations in [2]. Theorem 4 is proved by evaluating this quotient for certain specifically chosen functions $\Phi$. The restrictive hypothesis, that $\Lambda$ be an integral group ring, is only needed to ensure that $\Lambda$ is self-dual with respect to some suitable pairing.

Proofs of Theorems 1-4 will appear in [1]. 


\section{REFERENCES}

1. C. J. Bushnell and I. Reiner, Zeta functions of arithmetic orders and Solomon's conjectures (to appear).

2. R. Godement and H. Jacquet, Zeta-functions of simple algebras, Lecture Notes in Math., vol. 260, Springer-Verlag, Berlin, 1972.

3. L. Solomon, Zeta functions and integral representation theory, Advances in Math. 26 (1977), 306-326.

4. Partially ordered sets with colors, Amer. Math. Soc. Combinatorics Sympos., Columbus, Ohio, 1978.

DEPARTMENT OF MATHEMATICS, KING'S COLLEGE, UNIVERSITY OF LONDON, STRAND, LONDON WC 2R 2LS, ENGLAND

DEPARTMENT OF MATHEMATICS, UNIVERSITY OF ILLINOIS, URBANA, ILLINOIS 61801 